\title{
Room Temperature Magnetic Barrier Layers in Magnetic Tunnel Junctions
}

\author{
B. B. Nelson-Cheeseman, ${ }^{1, *}$ F. J. Wong, ${ }^{1}$ R. V. Chopdekar, ${ }^{1}$ E. Arenholz, ${ }^{2}$ and Y. Suzuki ${ }^{1}$ \\ ${ }^{1}$ Department of Materials Science and Engineering, University of California, Berkeley, CA 94720 \\ ${ }^{2}$ Advanced Light Source, Lawrence Berkeley National Laboratory, Berkeley, CA 94720
}

(Dated: June 3, 2010)

\begin{abstract}
We investigate the spin transport and interfacial magnetism of magnetic tunnel junctions with highly spin polarized LSMO and $\mathrm{Fe}_{3} \mathrm{O}_{4}$ electrodes and a ferrimagnetic $\mathrm{NiFe}_{2} \mathrm{O}_{4}$ (NFO) barrier layer. The spin dependent transport can be understood in terms of magnon-assisted spin dependent tunneling where the magnons are excited in the barrier layer itself. The $\mathrm{NFO} / \mathrm{Fe}_{3} \mathrm{O}_{4}$ interface displays strong magnetic coupling, while the LSMO/NFO interface exhibits clear decoupling as determined by a combination of X-ray absorption spectroscopy and X-ray magnetic circular dichroism. This decoupling allows for distinct parallel and antiparallel electrode states in this all-magnetic trilayer. The spin transport of these devices, dominated by the NFO barrier layer magnetism, leads to a symmetric bias dependence of the junction magnetoresistance at all temperatures.
\end{abstract}

PACS numbers:

\section{INTRODUCTION}

Magnetic tunnel junctions (MTJs) have been studied extensively as they are the potential building blocks of spin-based electronics. They are also excellent model systems for the study of magnetism at interfaces and spin injection. A MTJ device is composed of two ferromagnetic electrodes separated by an insulating barrier layer. The device exhibits a high or low resistance state depending on the relative orientation of the electrode magnetizations. ${ }^{1}$ In order to achieve distinct parallel and antiparallel resistance states to allow for two discrete junction resistance states, a non-magnetic insulating barrier layer is commonly employed, resulting in magnetically decoupled electrodes.

For many years, the spin polarization and spin scattering at the electrode-barrier interfaces was believed to be the largest determinant of spin-dependent transport in these MTJ devices, while the barrier layer was thought to act as a passive potential barrier to achieve the necessary spin-dependent tunneling and decouple the magnetic electrodes. However, recent investigations have shown that the choice of barrier layer material can have profound implications on the spin-based transport in these devices. This is particularly true for $\mathrm{Fe} / \mathrm{MgO} / \mathrm{Fe}$ MTJs where the $\mathrm{MgO}$ plays the symmetry filtering role, leading to highly polarized up-spin electrons to tunnel across the barrier. ${ }^{2,3}$ Recently, we demonstrated that inserting a paramagnetic barrier layer between magnetic electrodes does not preclude the observation of MTJ behavior. ${ }^{4}$

A completely ferromagnetic MTJ layer stack has yet to be demonstrated. The nature of spin transport in such MTJ's would provide insight into the role of the barrier layer and interfaces in such junction heterostructures. The effect of a ferromagnetic barrier layer on the magnetic coupling of the stack and the nature of the spin tunneling are of particular interest. The spinel ferrites, with strong exchange interactions and Curie temperatures well above room temperature, are excellent candidates to investigate as strongly magnetic barrier layer materials.
Additionally, they have recently proven to be successful magnetic barrier layer materials in spin filter tunnel junctions. ${ }^{5,6}$ Luders et al. saw significant junction magnetoresistance values at low temperatures in spin filter junctions of highly spin polarized $\mathrm{La}_{.7} \mathrm{Sr}_{.3} \mathrm{MnO}_{3}$ (LSMO) electrodes with $\mathrm{NiFe}_{2} \mathrm{O}_{4}$ (NFO) barrier layers and $\mathrm{Au}$ counter-electrodes. ${ }^{5}$ Ramos et al. were even able to attain small magnetoresistance values at room temperature in spin filter junctions with $\mathrm{CoFe}_{2} \mathrm{O}_{4}$ barrier layers. ${ }^{6}$

In this paper, we investigate the spin transport and magnetic coupling behavior of MTJ heterostructures with LSMO and $\mathrm{Fe}_{3} \mathrm{O}_{4}$ electrodes and ferrimagnetic $\mathrm{NiFe}_{2} \mathrm{O}_{4}$ barrier layers. Careful structural and magnetic characterization of the individual layers as well as the interfaces have been performed. We find that while the $\mathrm{NFO} / \mathrm{Fe}_{3} \mathrm{O}_{4}$ interface exhibits ferromagnetic coupling, the LSMO/NFO interface is magnetically decoupled. Therefore, in this all-magnetic stack, distinct parallel and antiparallel electrode states can be attained. Symmetric bias dependence of the junction magnetoresistance and inelastic tunneling spectra indicates that the spin transport of these devices is dominated by the barrier layer magnetism at all temperatures.

\section{EXPERIMENTAL METHODS}

Trilayers of $\operatorname{LSMO}(25 \mathrm{~nm}) / \mathrm{NFO}(3 \mathrm{~nm}) / \mathrm{Fe}_{3} \mathrm{O}_{4}(25 \mathrm{~nm})$ were grown by pulsed laser deposition on (110)-oriented $\mathrm{SrTiO}_{3}$ (STO) substrates. The (110) orientation creates an in-plane uniaxial magnetic anisotropy along the [001] direction for both the LSMO and $\mathrm{Fe}_{3} \mathrm{O}_{4}$. The LSMO was grown at $700^{\circ} \mathrm{C}$ in $300 \mathrm{mTorr}_{2}$; the $\mathrm{NFO}$ was grown at $550^{\circ} \mathrm{C}$ in 10 mTorr of a $99 \% \mathrm{~N}_{2} / 1 \% \mathrm{O}_{2}$ gaseous mixture; and the $\mathrm{Fe}_{3} \mathrm{O}_{4}$ was grown in a vacuum of $10^{-6}$ Torr at $400^{\circ} \mathrm{C}$. For these deposition conditions, single NFO films were insulating and magnetic at room temperature.

The epitaxy and crystallinity of the films on the STO substrate were investigated by X-ray diffraction and cross-sectional transmission electron microscopy (TEM) 
using a Philips CM 300 microscope at the National Center for Electron Microscopy, Lawrence Berkeley National Laboratory. The bulk magnetism of the heterostructures as a function of applied magnetic field was investigated by SQUID magnetometry. The interfacial chemistry and magnetism of the heterostructures was investigated by $\mathrm{Mn}$, Fe and Ni L-edge X-ray absorption spectroscopy (XAS) and X-ray magnetic circular dichroism (XMCD) on Beamline 6.3.1 at the Advanced Light Source (ALS). For XAS and XMCD studies, a $\mathrm{STO} / / \mathrm{LSMO} / \mathrm{NFO} / \mathrm{Fe}_{3} \mathrm{O}_{4}(0$ - $9 \mathrm{~nm}$ wedge $)$ trilayer sample was prepared whereby the graded thickness of the $\mathrm{Fe}_{3} \mathrm{O}_{4}$ film was achieved by the incremental movement of a Ta shutter over the sample during the $\mathrm{Fe}_{3} \mathrm{O}_{4}$ deposition. Due to the surface-sensitive XAS and XMCD in total electron yield mode (mean probe depth of $5 \mathrm{~nm}$ ), this wedge sample geometry enables different depths of the heterostructure to be probed. The MTJs were fabricated by conventional optical lithography and Ar ion milling, and ranged in size from $4 \times 4-40 \mathrm{x} 40 \mu \mathrm{m}^{2}$. Transport measurements were conducted in magnetic fields up to $8 \mathrm{kOe}$ and temperatures from 30 to $300 \mathrm{~K}$. The junction magnetoresistance (JMR) was calculated using Julliere's model by the following equation: $\operatorname{JMR}(\%)=\left(\mathrm{R}_{A P^{-}}\right.$ $\left.\mathrm{R}_{P}\right) / \mathrm{R}_{P}{ }^{*} 100$ where $\mathrm{R}_{P}=\mathrm{R}(\mathrm{H}=0$ Oe $)$.

\section{STRUCTURAL PROPERTIES}

\section{A. X-ray Diffraction}

The crystallinity and epitaxy of the trilayer samples were investigated by $\mathrm{Cu} \mathrm{K}_{\alpha}$ X-ray diffraction. Specular X-ray diffraction of the unpatterned trilayers shows only $\{110\}$ reflection peaks from the perovskite and spinel crystal structures, indicating epitaxy with the STO substrate. Single phase perovskite LSMO and spinel $\mathrm{Fe}_{3} \mathrm{O}_{4}$ crystal structures are confirmed. The ultrathin NFO film peaks are likely obscured by the stronger peaks from the thicker $\mathrm{Fe}_{3} \mathrm{O}_{4}$ film.

\section{B. Transmission Electron Microscopy}

The crystallinity and epitaxy of the films and interfaces were also investigated by cross-sectional TEM. As seen in Figure 1, excellent crystallinity of both the perovskite and spinel crystal structures is seen by high resolution transmission electron microscopy (HR-TEM) and the accompanying Fast Fourier Transform (FFT). One can see that the spinel films grow epitaxially and with high crystallinity on the perovskite LSMO template and that a relatively abrupt interface exists between the two crystal structures. The presence of two sets of spots in the FFT indicates that the spinel-structured films are relaxed on the perovskite template. This is likely enabled by misfit dislocations and other defects in the

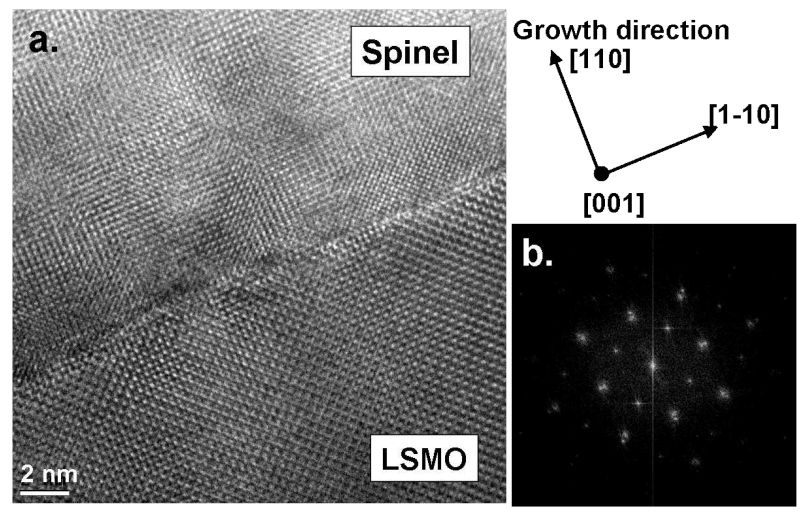

FIG. 1: Cross-sectional HR-TEM of LSMO/NFO(3 $\mathrm{nm}) / \mathrm{Fe}_{3} \mathrm{O}_{4}$ trilayer showing high crystallinity and epitaxy of the LSMO, NFO and $\mathrm{Fe}_{3} \mathrm{O}_{4}$ films. The LSMO/NFO interface is characterized by defects within the spinel structure, while the $\mathrm{NFO} / \mathrm{Fe}_{3} \mathrm{O}_{4}$ interface cannot be seen, indicating a coherent interface.

spinel at the interface. Although a small amount of interfacial defects in the spinel structure arise at the perovskite/spinel interface, these defects allow the spinel films to maintain good structural registry with the perovskite underlayer. ${ }^{8}$ Meanwhile, there is no distinct contrast of the spinel/spinel interface in the HR-TEM image, strongly suggesting that the $\mathrm{NFO} / \mathrm{Fe}_{3} \mathrm{O}_{4}$ interface is coherent.

\section{CHEMICAL PROPERTIES}

\section{A. X-ray Absorption Spectroscopy}

The chemical order at and near the interfaces of the heterostructure was studied by XAS. The valence states of the $\mathrm{Ni}, \mathrm{Mn}$ and Fe cations near the LSMO/NFO and $\mathrm{NFO} / \mathrm{Fe}_{3} \mathrm{O}_{4}$ interfaces were probed by L-edge XAS of the $\mathrm{STO} / / \mathrm{LSMO}(25 \mathrm{~nm}) / \mathrm{NFO}(3 \mathrm{~nm}) / \mathrm{Fe}_{3} \mathrm{O}_{4}$ (wedge) trilayer. The surface-sensitivity of L-edge XAS in total electron yield mode allows one to selectively probe the chemical signatures near the two interfaces by probing at various points along the wedge sample.

First we examine the isostructural $\mathrm{NFO} / \mathrm{Fe}_{3} \mathrm{O}_{4}$ interface. As seen in Figure 2, the Ni lineshape is indicative of $\mathrm{Ni}^{2+}$ and does not change across the sample. ${ }^{9}$ This indicates that the $\mathrm{Ni}$ remains $\mathrm{Ni}^{2+}$ throughout the NFO film and at the interface. The extra peak at $852 \mathrm{eV}$ corresponds to the La M-edge, and arises from the La of the LSMO bottom layer. Note that the Ni total electron yield signal decreases by over an order of magnitude across the wedge sample causing background effects to become more prominent for positions $\mathrm{C}$ and $\mathrm{D}$. The shoulder in the $\mathrm{Fe}$ XAS at $708 \mathrm{eV}$ changes slightly across the wedge. This feature is commonly associated with a change in Fe valence and site occupancy. ${ }^{10}$ Thus, this change is expected as NFO has only $\mathrm{Fe}^{3+}$ cations, while $\mathrm{Fe}_{3} \mathrm{O}_{4}$ contains $\mathrm{Fe}^{2+}$ 


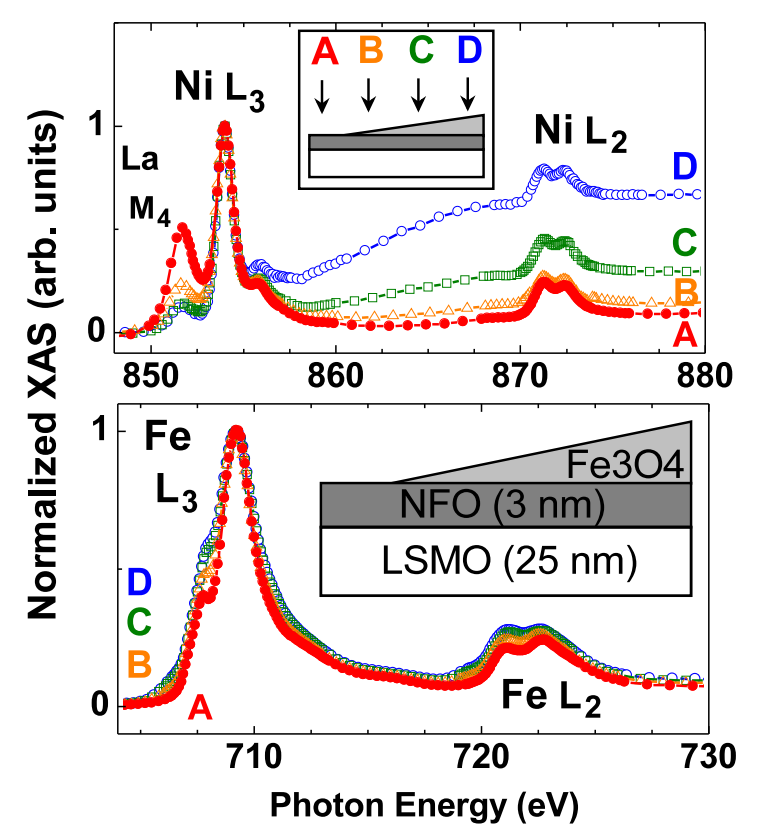

FIG. 2: (Color Online.) Normalized L-edge XAS of $\mathrm{NFO} / \mathrm{Fe}_{3} \mathrm{O}_{4}$ interface at various positions along the trilayer wedge sample, as indicated.

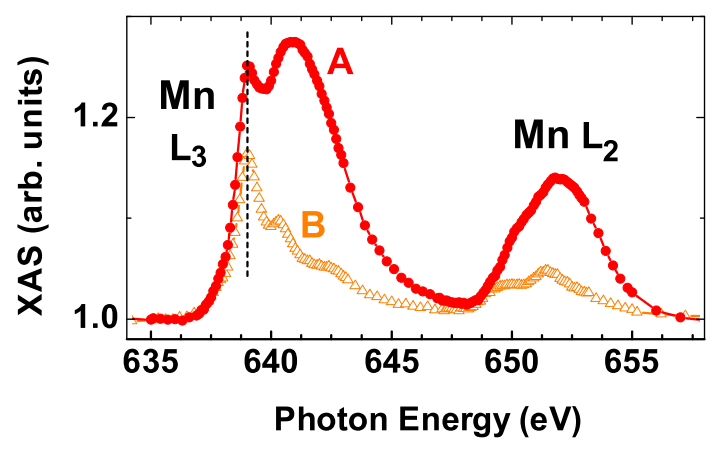

FIG. 3: (Color Online.) Mn L-edge XAS of LSMO/NFO interface at positions $\mathrm{A}$ and $\mathrm{B}$ along trilayer wedge sample, showing presence of $\mathrm{Mn}^{2+}$ near interface.

and $\mathrm{Fe}^{3+}$ cations.

Now we examine the non-isostructural LSMO/NFO interface. As already seen in Figure 2, the $\mathrm{Ni}$ and $\mathrm{Fe} \mathrm{L}$ edge XAS at positions A and B closely resemble those of NFO single films. ${ }^{9,10}$ The Mn L-edge XAS at positions $\mathrm{A}$ and $\mathrm{B}$ should correspond to $\mathrm{Mn}^{3+}$ and $\mathrm{Mn}^{4+}$ in the LSMO layer. The Mn L-edge spectra for position A is shown in Figure 3. At higher energies the spectra mimics the typical LSMO spectra seen in single films, while the presence of the small peak at $638.9 \mathrm{eV}$ does not. ${ }^{11}$ This feature on the low photon energy side of the $\mathrm{Mn} \mathrm{L}_{3}$

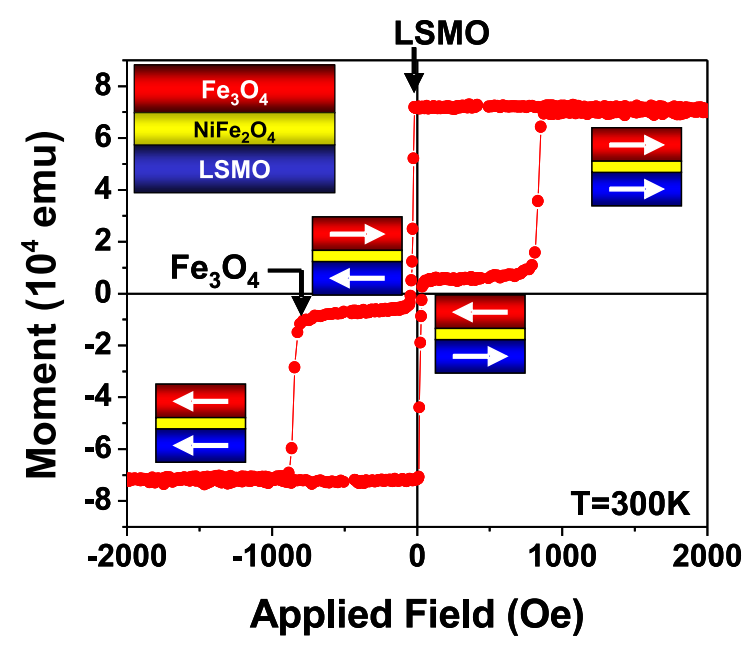

FIG. 4: (Color Online.)Bulk moment of a $\mathrm{Fe}_{3} \mathrm{O}_{4} / \mathrm{NFO} / \mathrm{LSMO}$ trilayer as a function of applied field at $300 \mathrm{~K}$, showing creation of distinct parallel and antiparallel magnetization states.

absorption edge has been associated with the presence of either $\mathrm{Mn}^{2+}, \mathrm{Mn}^{4+}$, or a changing symmetry of the $\mathrm{Mn}$ environment. ${ }^{11-13}$

In order to determine the source of the peak at 638.9 $\mathrm{eV}$, the spectrum at position $\mathrm{B}$ was analyzed and found to correspond to $\mathrm{Mn}^{2+}$ in a $\mathrm{MnFe}_{2} \mathrm{O}_{4}$-environment (Figure 3). ${ }^{14}$ While position A corresponds to no $\mathrm{Fe}_{3} \mathrm{O}_{4}$ top layer, position $\mathrm{B}$ corresponds to a top $\mathrm{Fe}_{3} \mathrm{O}_{4}$ layer of 1-2 $\mathrm{nm}$. Therefore, the spectrum at $\mathrm{B}$ picks up changes induced in the Mn by and near the additional $\mathrm{NFO} / \mathrm{Fe}_{3} \mathrm{O}_{4}$ interface. Together these observations suggest that the Mn near the LSMO/NFO interface (possibly in the NFO barrier or at the $\mathrm{NFO} / \mathrm{Fe}_{3} \mathrm{O}_{4}$ interface) has a valence of $2+$ and is in a $\mathrm{MnFe}_{2} \mathrm{O}_{4}$-environment.

\section{MAGNETIC PROPERTIES}

\section{A. Bulk Magnetism}

The bulk magnetism of the unpatterned trilayers as a function of applied magnetic field was investigated by SQUID magnetometry. As seen in Figure 4, we obtain abrupt magnetic switching of the $\mathrm{LSMO}$ and $\mathrm{Fe}_{3} \mathrm{O}_{4}$ electrodes at $300 \mathrm{~K}$. Large coercive field differences between the LSMO and $\mathrm{Fe}_{3} \mathrm{O}_{4}$ create well-defined parallel and antiparallel magnetization states at all temperatures, even though the NFO barrier layer is ferrimagnetic at all temperatures measured. The NFO film magnetism cannot be separated in this hysteresis loop since it represents a small moment compared to those of the thick electrode materials. Nevertheless, it is striking that we obtain distinct switching between the antiparallel and parallel electrode magnetization states in this all-magnetic stack. 


\section{B. Interface Magnetism}

In order to elucidate the magnetic interactions among the LSMO, NFO and $\mathrm{Fe}_{3} \mathrm{O}_{4}$ layers in this allmagnetic stack, we performed $\mathrm{Mn}, \mathrm{Fe}$ and $\mathrm{Ni}$ L-edge $\mathrm{XMCD}$ of the $\mathrm{STO} / / \mathrm{LSMO} / \mathrm{NFO} / \mathrm{Fe}_{3} \mathrm{O}_{4}$ (wedge) trilayer (Figure5(insets).) The surface-sensitivity of XMCD in total electron yield mode allows one to selectively probe the magnetic phenomena at and near the two interfaces by probing at various points along the wedge sample. All investigations were done at $300 \mathrm{~K}$.

First we examine the $\mathrm{NFO} / \mathrm{Fe}_{3} \mathrm{O}_{4}$ interface. The $\mathrm{XMCD}$ investigations of the trilayer wedge show that the $\mathrm{NFO}$ and $\mathrm{Fe}_{3} \mathrm{O}_{4}$ layers of the heterostructure display XMCD spectra characteristic of single films of NFO and $\mathrm{Fe}_{3} \mathrm{O}_{4}$, respectively. The $\mathrm{Ni}$ and $\mathrm{Fe}$ XMCD spectra are displayed in Figure 5. The exposed NFO on LSMO with no $\mathrm{Fe}_{3} \mathrm{O}_{4}$ top layer (A) shows $\mathrm{Ni}$ and $\mathrm{Fe}$ dichroism characteristic of a single $\mathrm{NiFe}_{2} \mathrm{O}_{4}$ thin film, while the thick $\mathrm{Fe}_{3} \mathrm{O}_{4}$ top layer on NFO (D) exhibits Fe dichroism characteristic of a single $\mathrm{Fe}_{3} \mathrm{O}_{4}$ film. ${ }^{9,15}$ It is interesting to note that although the Ni XAS intensity decreases substantially with increasing $\mathrm{Fe}_{3} \mathrm{O}_{4}$ overlay thickness across the wedge from position A to D (Figure 2), the Ni XMCD signal increases using the Ni X-ray absorption $\mathrm{L}_{3}$ edge for normalization. It appears that proximity effects at the spinel-spinel interface enhances the moment alignment of the $\mathrm{Ni}^{2+}$ cations.

The coincident $\mathrm{Ni}(854.1 \mathrm{eV})$ and Fe $(707.7 \mathrm{eV})$ hysteresis loops taken at all positions along the wedge demonstrate strong interfacial ferromagnetic coupling between the NFO and $\mathrm{Fe}_{3} \mathrm{O}_{4}$ layers (Figure 6.) While this is expected when only the NFO thin film is being probed (A), the coincidence in $\mathrm{Ni}$ and Fe hysteresis loops persists even when the Fe signal is dominated by the $\mathrm{Fe}_{3} \mathrm{O}_{4}$ top layer (D). The coincidence of the $\mathrm{Ni}$ and $\mathrm{Fe}$ loops across the wedge sample confirms that the $\mathrm{NFO}$ and $\mathrm{Fe}_{3} \mathrm{O}_{4}$ films are ferromagnetically coupled across the spinel/spinel interface.

A detailed investigation of the LSMO/NFO interface indicates that there is cation intermixing. As seen in Figure 7, the Mn XMCD spectra changes quite dramatically in the presence of the $\mathrm{NFO} / \mathrm{Fe}_{3} \mathrm{O}_{4}$ interface. At position $\mathrm{A}$, we see that the Mn XMCD spectra closely resembles that of single LSMO films. ${ }^{16}$ At position B, Figures 3 and 7 show that both the Mn XAS and XMCD spectra closely resemble that of $\mathrm{Mn}$ in $\mathrm{MnFe}_{2} \mathrm{O}_{4} \cdot{ }^{14}$ These Mn XAS and XMCD results can be explained by a small amount of $\mathrm{Mn}$ cation mixing from the perovskite into the tetrahedral sites of the spinel near the LSMO/NFO interface.

Surprisingly, although there is cation intermixing at the LSMO/NFO interface, the neighboring spinel and perovskite layers are magnetically decoupled. The element-specific hysteresis loops for position A are shown in Figure 8(a). The Mn shows abrupt magnetic switching at low fields, characteristic of single LSMO films, while the Ni and Fe show hysteretic behavior distinct from that

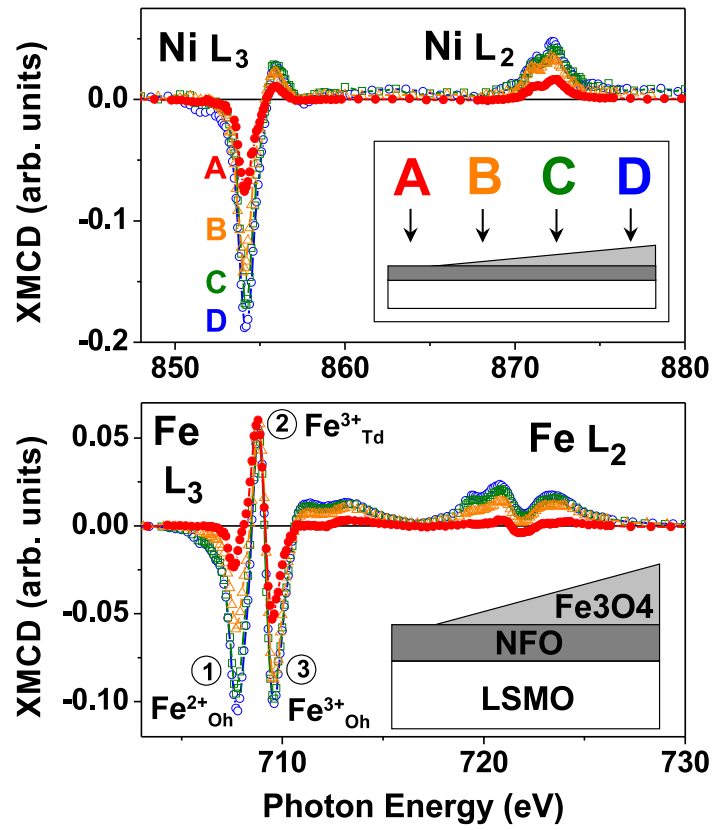

FIG. 5: (Color Online.) Ni and Fe XMCD spectra using +/$1.5 \mathrm{~T}$ from various positions across wedge, as indicated.

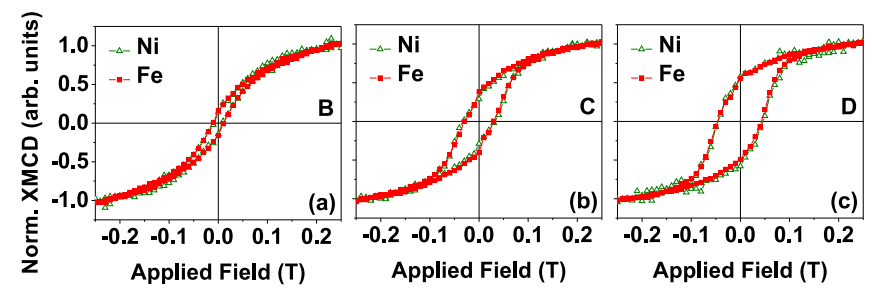

FIG. 6: (Color Online). Ni (854.1 eV) and Fe (707.7 eV) hysteresis loops across trilayer wedge demonstrating coincident switching.

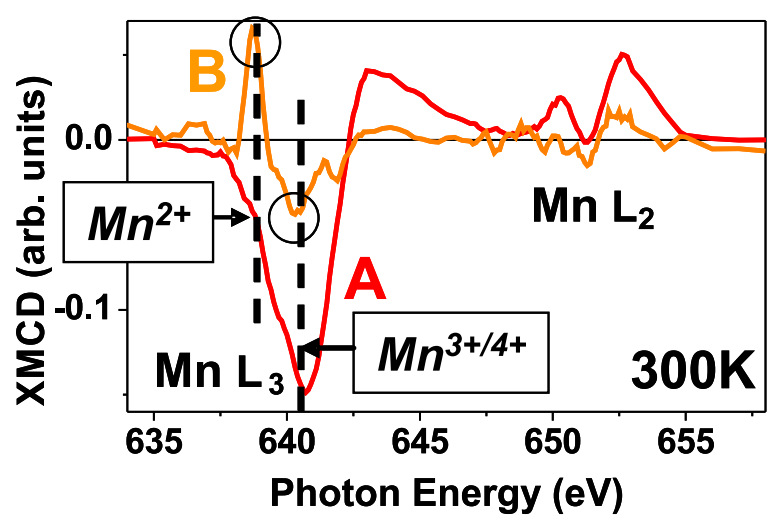

FIG. 7: (Color Online). Mn XMCD using +/-1.5 T at positions $\mathrm{A}$ and $\mathrm{B}$ exhibit different lineshapes. 


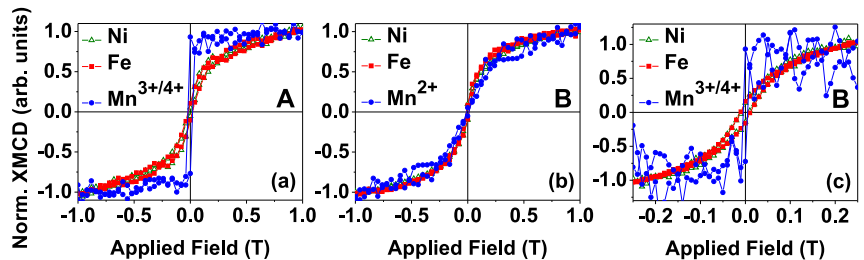

FIG. 8: (Color Online). Mn, Fe and Ni XMCD hysteresis loops taken at (a) position $\mathrm{A}$ and (b-c) position $\mathrm{B}$.

of the Mn. The XAS, XMCD and hysteresis behavior seen at position $\mathrm{A}$ is also reproduced in LSMO/NFO bilayer samples (not shown). Together this indicates independent magnetic switching between the perovskite LSMO and spinel NFO layers.

At position $\mathrm{B}$, the $\mathrm{Mn}$ displays different magnetic switching behavior as a function of valence state. Hysteresis loops were taken at two different photon energies of the $\mathrm{Mn} \mathrm{L}_{3}$-edge, as circled and labeled in Figure 7. The resulting hysteresis loops are shown in Figure 8(b-c) and overlaid with the $\mathrm{Ni}$ and $\mathrm{Fe}$ hysteresis loops from the same location on the sample (B). One can see that the hysteresis loop probed at the characteristic $\mathrm{Mn}^{2+}$ energy mimics the $\mathrm{Ni}$ and Fe hysteresis loops, again indicating that there is some Mn incorporated into NFO creating a mixed $(\mathrm{Ni}, \mathrm{Fe}, \mathrm{Mn})_{3} \mathrm{O}_{4}$. Nevertheless, the field-dependent Mn XMCD measured at the characteristic $\mathrm{Mn}^{3+/ 4+}$ energy still shows abrupt switching at low fields independent of the $\mathrm{Ni}$ and $\mathrm{Fe}$. The presence of these distinct $\mathrm{Mn}$ hysteresis loops taken from the same exact location on the sample underlines the robust and abrupt magnetic decoupling between the spinel and perovskite materials.

\section{MAGNETOTRANSPORT}

\section{A. Magnetic Field Dependence}

The behavior of the junction resistance was first investigated as a function of applied magnetic field. One can see from Figure 9 that the junction resistance switches abruptly between a high resistance and a low resistance state. This abrupt switching is maintained at all measurement temperatures $(30-300 \mathrm{~K})$ and is consistent with magnetic decoupling of the LSMO and NFO. The junctions yield JMR values of up to $-14 \%$ at $80 \mathrm{~K}$. The antiparallel $\mathrm{LSMO}-\mathrm{Fe}_{3} \mathrm{O}_{4}$ magnetization configuration is the low resistance state, which results in a negative magnetoresistance. This behavior can be explained in terms of opposite signs of the LSMO and $\mathrm{Fe}_{3} \mathrm{O}_{4}$ electrode spin polarizations; LSMO is positive and $\mathrm{Fe}_{3} \mathrm{O}_{4}$ is negative. ${ }^{17}$

Here we see that even in the presence of a magnetic barrier layer this all-magnetic stack still exhibits very distinct parallel and antiparallel resistance states due to the magnetic decoupling at the LSMO-NFO perovskitespinel interface. Thus, while the epitaxial growth of

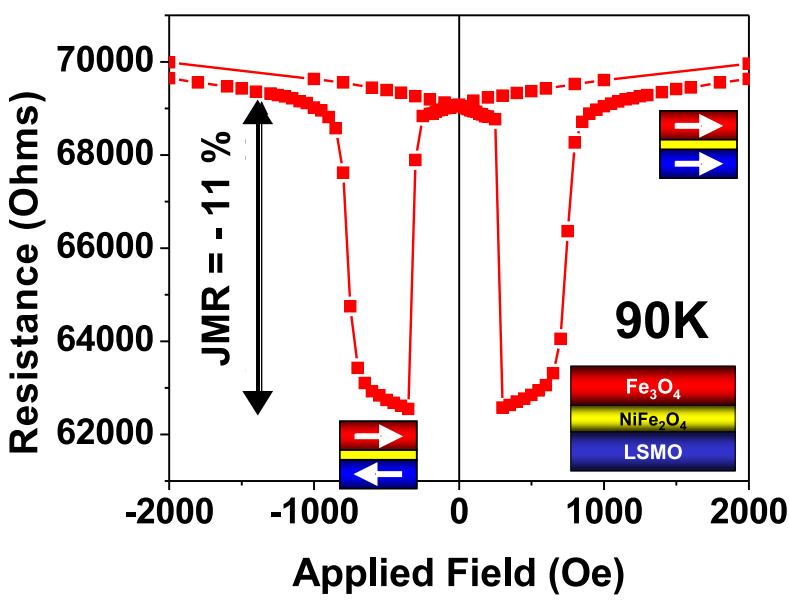

FIG. 9: (Color Online). Junction resistance and JMR as a function of applied magnetic field.

LSMO and $\mathrm{Fe}_{3} \mathrm{O}_{4}$ in these heterostructures is important for MTJ applications due to the desired coincident in-plane magnetic easy axes it brings, the nonisostructural yet epitaxial perovskite/spinel interface allows for distinct magnetic decoupling between adjacent magnetic layers. Such behavior may likely be a general phenomenon of perovskite/spinel interfaces, and proves promising for the exploration of other decoupled magnetic barrier/electrode combinations for spintronic devices.

\section{B. Temperature Dependence}

The temperature dependence of the JMR was investigated in these junctions to determine the effect of a strongly magnetic barrier layer on the spin transport. The temperature dependence of the JMR is shown in Figure 10. We see that the JMR magnitude increases with decreasing temperature between $200 \mathrm{~K}$ and $80 \mathrm{~K}$. A monotonic increase in JMR with decreasing temperature is common for oxide MTJ devices. ${ }^{18}$ However, below $80 \mathrm{~K}$, the JMR magnitude begins to decrease with decreasing temperature. At these temperatures, the total junction resistance increases markedly and the resistance difference due to the parallel and antiparallel resistance states progressively becomes a decreasing percentage of the total resistance (Figure 10.)

This is expected from the temperature-dependence of the resistivity of $\mathrm{Fe}_{3} \mathrm{O}_{4} \cdot{ }^{19}$ As has been explained in similar $\mathrm{Fe}_{3} \mathrm{O}_{4}$-based junctions, ${ }^{4,20}$ we attribute this decrease in JMR at low temperatures to a suppressed Verwey metal-insulator transition of the $\mathrm{Fe}_{3} \mathrm{O}_{4}$ thin film electrode, which is consistent with the temperature dependence of the junction resistance. While bulk $\mathrm{Fe}_{3} \mathrm{O}_{4}$ undergoes this transition at $120 \mathrm{~K}$, it is common for epi- 


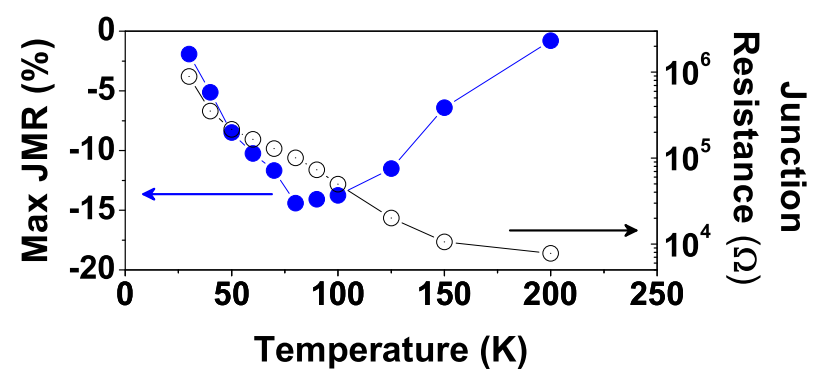

FIG. 10: (Color Online). Maximum JMR as a function of temperature for $\mathrm{LSMO} / \mathrm{NFO} / \mathrm{Fe} 3 \mathrm{O} 4$ junctions.

taxial $\mathrm{Fe}_{3} \mathrm{O}_{4}$ films to show a more suppressed Verwey transition with less abrupt changes in resistivity. This suppression has been attributed to defects in the films, such as antiphase boundaries. ${ }^{21}$

The disappearance of the JMR by $200 \mathrm{~K}$ seen in our NFO junctions is attributed in large part to the LSMO electrode. It has been widely seen that junctions incorporating LSMO electrode materials exhibit a strong temperature-dependent decrease in JMR values, which is attributed to degradation of the LSMO spin polarization at the electrode-barrier interface. ${ }^{5,17,20}$ Furthermore, the Mn cation migration evidenced in the XAS and XMCD results may deplete the LSMO of Mn near the interface, which would in turn depress the interfacial spin polarization. Luders et al. found that the JMR values of their LSMO/NFO spin filter junctions also vanished by $200 \mathrm{~K}$, again pointing to characteristics of the LSMO/NFO interface as a limiting factor in attaining room temperature magnetoresistance values. ${ }^{5}$ Such interfacial Mn cation migration effects could also be the reason for the overall lower JMR values of the NFO junctions compared to the paramagnetic NMO junctions previously studied. ${ }^{4}$ It is consistent with Mn already present in the NMO barrier layer, resulting in a smaller driving force for $\mathrm{Mn}$ of the LSMO to migrate across the interface into the spinelstructure barrier layer, resulting in larger LSMO interfacial spin polarization values and higher JMR values for a given temperature.

In addition to the degradation of the LSMO interfacial spin polarization, another possible factor contributing to the disappearance of JMR at $200 \mathrm{~K}$ is the barrier height. Ramos et al. found that the lack of full oxygenation of $\mathrm{CoFe}_{2} \mathrm{O}_{4}$ barrier layers leads to barrier lowering, thus lowering the JMR values of such spin filter junctions. ${ }^{22}$ This is also supported by the linear current-voltage characteristics seen in our NFO junctions beginning around $200 \mathrm{~K}$, indicating loss of an effective potential barrier. The NFO barrier layer was grown in oxygen-poor conditions and exposed to vacuum at $400{ }^{\circ} \mathrm{C}$ in order to avoid formation of $\mathrm{Fe}_{2} \mathrm{O}_{3}$ during the growth of the $\mathrm{Fe}_{3} \mathrm{O}_{4}$ top electrode. This is a technical obstacle which may be overcome by selection of the right barrier/electrode materials

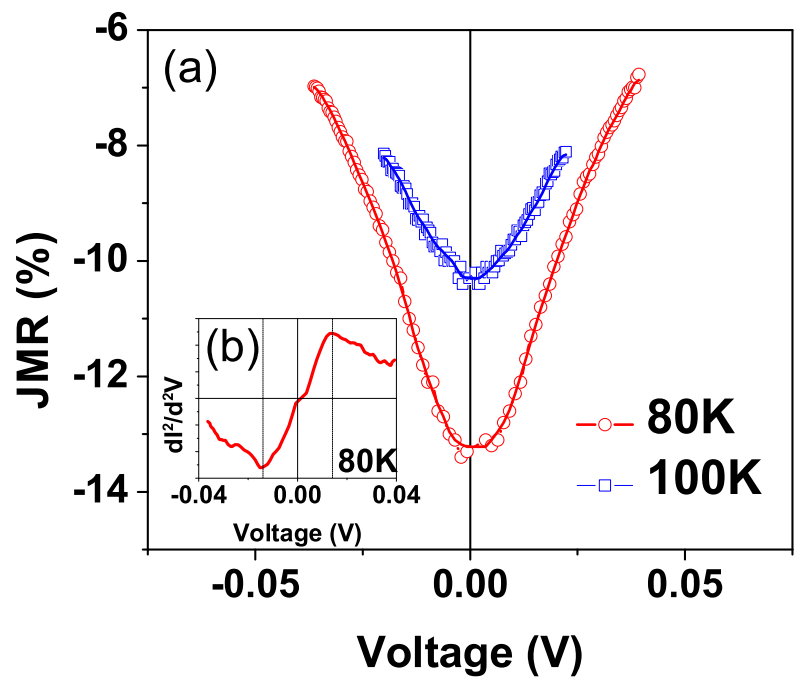

FIG. 11: (Color Online). Bias dependence of magnetotransport for $\mathrm{LSMO} / \mathrm{NFO} / \mathrm{Fe}_{3} \mathrm{O}_{4}$ junctions. Voltage dependence of (a) JMR and (b) IETS are symmetric with respect to $0 \mathrm{~V}$.

combination.

\section{Bias Dependence}

Finally, we investigate the bias dependence of the magnetotransport using a 4-point probe geometry. Conductance curves of the junctions were measured and fit to direct tunneling at low biases and to hopping through two or more states at higher bias. This behavior reflects the importance of defect-mediated transport in these junctions at higher bias.

The JMR displays a symmetric monotonic bias dependence for all temperatures, characterized by a maximum JMR at zero bias and a dramatic drop off of JMR with increasing bias, as shown in Figure 11(a). Magnetic tunnel junctions commonly have asymmetric bias dependencies based on the dissimilar electrode/barrier band offsets and density of states. The symmetric bias dependence seen in these junctions indicate that the barrier itself is dominating the spin transport regardless of the direction bias is applied. Symmetric bias dependence of the JMR has also been seen in junctions with $\mathrm{BiMnO}_{3}{ }^{23}$ and $\mathrm{NiMn}_{2} \mathrm{O}_{4}{ }^{4}$ barrier layers when they exhibit long-range magnetism. This symmetry is a signature that the barrier layer and not the electrode/barrier interfaces dominate the spin transport in these NFO devices. ${ }^{4,24}$

The bias symmetry of the JMR curves indicate that the barrier layer is not just a passive layer through which electrons directly tunnel. In order to investigate the role of magnons from the magnetic barrier in our junctions, we plot the second derivative of the current-voltage curves, known as the inelastic tunneling spectra (IETS). The 
IETS illustrates the inelastic tunneling processes due to phonons and magnons. In order to isolate the effects of magnetism on the inelastic transport, IETS from the two distinct magnetization configurations $\left(\operatorname{IETS}_{P}\right.$ and IETS $\left._{A P}\right)$ are subtracted from one another. The resulting IETS shows inelastic tunneling events related solely to magnons as all other factors are held constant between both magnetization configurations. ${ }^{24}$ As shown in Figure 11(b), the subtracted IETS shows symmetry of the peaks at positive and negative voltage, and the peak locations $(+/-14 \mathrm{mV}$ at $80 \mathrm{~K})$ are well within the range of $12-100 \mathrm{mV}$ commonly associated with magnons. ${ }^{25,26}$ The characteristic magnon-assisted transport across the heterostructures of $14 \mathrm{mV}$ corresponds to $180 \mathrm{~K}$, and may explain why the IV curves become linear and JMR is lost above 200K. The symmetric nature of the IETS indicates that almost identical magnon-assisted tunneling events dominate the conduction regardless of the direction of applied bias. This again points to the dominant role of magnon-mediated transport through the magnetic barrier layer. It is quite remarkable that despite such hopping transport through the magnetic barrier, there are very little spin flip scattering events that would destroy the distinct $\mathrm{P}$ and AP states observed in the JMR.

\section{CONCLUSIONS}

In conclusion, we have studied the magnetic coupling behavior and spin transport of magnetic tunnel junction heterostructures with highly spin polarized electrodes and room temperature ferrimagnetic $\mathrm{NiFe}_{2} \mathrm{O}_{4}$ barrier layers. We see that introducing a room temperature ferrimagnetic barrier layer into these novel allmagnetic device heterostructures does not preclude the abrupt switching of the electrodes between parallel and antiparallel magnetization and high and low resistance states. This is due to the robust magnetic decoupling seen between the perovskite and spinel crystal structures at the non-isostructural LSMO/NFO interface. However, we note that there is limited cation migration at the perovskite/spinel interface, which must be considered when utilizing such interfaces in magnetic devices. The interfacial chemistry at transition metal spinel and perovskite interfaces remains a vast field to be explored by XAS and XMCD. The spin transport of these devices is dominated by the barrier layer magnetism, which leads to a symmetric bias dependence of the JMR at all temperatures. The decrease of JMR to zero by $200 \mathrm{~K}$ is attributed to the degradation of the interfacial spin polarization of the LSMO electrode, and barrier lowering of the NFO barrier layer. The investigation of alternate electrode and barrier layer materials will provide further understanding of this new all-magnetic heterostructure for MTJ applications.

VIII. ACKNOWLEDGEMENTS

This work was supported through the Materials Science Division and Advanced Light Source by the Director, Office of Science, Office of Basic Energy Sciences, Division of Materials Sciences and Engineering, of the U.S. Department of Energy under Contract No. DEAC02-05CH11231. RVC was supported by NSF Division of Materials Research Fund 06. Portions of this work were performed at the National Center for Electron Microscopy, Lawrence Berkeley National Laboratory. Device processing was performed at the University of California - Berkeley Microlab. BBNC would like to acknowledge the support of fellowships from NSF-IGERT and the Intel Foundation.
* Electronic address: bbnelsonchee@berkeley.edu

1 J.S. Moodera, J. Nassar and G. Mathon. Ann. Rev. Mat. Sci. 29, 381 (1999).

2 W. H. Butler, X. -G. Zhang, T. C. Schulthess, and J. M. MacLaren, Phys. Rev. B 63, 054416 (2001).

3 J. Mathon and A. Umerski. Phys. Rev. B 63, 220403(R) (2001).

4 B.B. Nelson-Cheeseman, R. V. Chopdekar, L. M. B. Alldredge, J.S. Bettinger, E. Arenholz, and Y. Suzuki. Phys. Rev. B 76, 220410(R) (2007).

5 U. Luders, M. Bibes, K. Bouzehouane, E. Jacquet, J.P. Contour, S. Fusil, J.-F. Bobo, J. Fontcuberta, A. Barthelemy, and A. Fert. Appl. Phys. Lett. 88, 082505 (2006).

6 A. V. Ramos, M.-J. Guittet, J.-B. Moussy, R. Mattana, C. Deranlot, F. Petroff, and C. Gatel. Appl. Phys. Lett. 91, 122107 (2007).

7 M. Julliere, Phys. Lett. A. 54, 225 (1975).

8 B. B. Nelson-Cheeseman, F. Wong, R. V. Chopdekar, M. Chi, E. Arenholz, N. D. Browning, and Y. Suzuki. J. Vac. Sci. Technol. B 26, 1521 (2008).
${ }^{9}$ G. van der Laan, C. M. B. Henderson, R. A. D. Pattrick, S. S. Dhesi, P. F. Schofield, E. Dudzik, and D. L. Vaughan. Phys. Rev. B. 59, 4314 (1999).

10 W.F. Pong, Y.K. Chang, M.H. Su, P.K. Tseng, H.J. Lin, G.H. Ho, K.L. Tsang, and C.T. Chen. Phys. Rev. B. 55, 11409 (1997).

11 R. V. Chopdekar, E. Arenholz, and Y. Suzuki, Phys. Rev. B. 79, 104417 (2009).

12 M. P. de Jong, I. Bergenti, V. A. Dediu, M Fahlman, M. Marsi, and C. Taliani. Phys. Rev. B. 71, 014434 (2005).

13 B. Gilbert, B. H. Frazer, A. Belz, P. G. Conrad, K. H. Nealson, D. Haskel, J. C. Land, G. Srajer, and G. De Stasio. J. Phys. Chem. A. 107, 2839 (2003).

14 J.-S. Kang, G. Kim, H.J. Lee, D.H. Kim, H.S. Kim, J.H. Shim, S. Lee, H.G. Lee, J.-Y. Kim, B.H. Kim, and B.I. Min. Phys. Rev. B. 77, 035121 (2008).

15 R.A.D. Pattrick, G. van der Laan, C.M.B. Henderson, P. Kuiper, E. Dudzik, D.J. Vaughan. Eur. J. Mineral. 14, 1095 (2002).

16 R. Stadler, Y.U. Idzerda, Z. Chen, S.B. Ogale, T. Venkatesan, Appl. Phys. Lett. 75, 3384 (1999). 
17 G. Hu and Y. Suzuki, Phys. Rev. Lett. 89, 276601 (2002).

18 M. Bibes and A. Barthelemy. IEEE Transactions on Electron Devices. 54, 1003 (2007).

19 E.J.W. Verwey, P.W. Haayman, Physica 8, 979 (1941).

20 L.M.B.Alldredge, R.V.Chopdekar, B.Nelson-Cheeseman, Y. Suzuki, Appl. Phys. Lett. 89, 182504 (2006).

21 M. G. Chapline and S. X. Wang, J. Appl. Phys. 97, 123901 (2005).

22 A. V. Ramos, T. S. Santos, G. X. Miao, M.-J. Guittet, J.-B. Moussy, and J. S. Moodera, Phys. Rev. B. 78, 180402(R) (2008).

23 M. Gajek, M. Bibes, A. Barthelemy, K. Bouzehouane, S. Fusil, M. Varela, J. Fontcuberta, A. Fert, Phys. Rev. B 72, 020406(R) (2005).

24 J.S. Moodera, J. Nowak, R.J.M. van de Veerdonk, Phys. Rev. Lett. 80, 2941 (1998).
25 D.C. Tsui, R.E. Dietz, L.R. Walker, Phys. Rev. Lett. 27, 1729 (1971).

26 Y. Ando, J. Murai, H. Kubota, and T. Miyazaki, J. Appl. Phys. 87, 5209 (2000)

27 J.S. Moodera, X. Hao, G.A. Gibson, and R. Meservey. Phys. Rev. Lett. 61, 637 (1988).

28 X. Hao, J.S. Moodera, and R. Meservey. Phys. Rev. B. 42, 8235 (1990).

29 J.S. Moodera, R. Meservey and X. Hao. Phys. Rev. Lett. 70, 853 (1993).

30 T. Nagahama, T.S. Santos and J.S. Moodera. Phys. Rev. Lett. 99, 016602 (2007).

31 J.S. Moodera, T.S. Santos and T. Nagahama. J. Phys.: Condens. Matter. 19, 165202 (2007). 